\title{
Grey matter abnormalities are associated only with severe cognitive decline in early stages of Parkinson's disease
}

Citation for published version (APA):

Wolters, A. F., Moonen, A. J. H., Lopes, R., Leentjens, A. F. G., Duits, A. A., Defebvre, L., Delmaire, C., Hofman, P. A., van Bussel, F. C., \& Dujardin, K. (2020). Grey matter abnormalities are associated only with severe cognitive decline in early stages of Parkinson's disease. Cortex, 123, 1-11. https://doi.org/10.1016/j.cortex.2019.09.015

Document status and date:

Published: 01/02/2020

DOI:

10.1016/j.cortex.2019.09.015

Document Version:

Publisher's PDF, also known as Version of record

\section{Document license:}

Taverne

Please check the document version of this publication:

- A submitted manuscript is the version of the article upon submission and before peer-review. There can be important differences between the submitted version and the official published version of record.

People interested in the research are advised to contact the author for the final version of the publication, or visit the DOI to the publisher's website.

- The final author version and the galley proof are versions of the publication after peer review.

- The final published version features the final layout of the paper including the volume, issue and page numbers.

Link to publication

\footnotetext{
General rights rights.

- You may freely distribute the URL identifying the publication in the public portal. please follow below link for the End User Agreement:

www.umlib.nl/taverne-license

Take down policy

If you believe that this document breaches copyright please contact us at:

repository@maastrichtuniversity.nl

providing details and we will investigate your claim.
}

Copyright and moral rights for the publications made accessible in the public portal are retained by the authors and/or other copyright owners and it is a condition of accessing publications that users recognise and abide by the legal requirements associated with these

- Users may download and print one copy of any publication from the public portal for the purpose of private study or research.

- You may not further distribute the material or use it for any profit-making activity or commercial gain

If the publication is distributed under the terms of Article $25 \mathrm{fa}$ of the Dutch Copyright Act, indicated by the "Taverne" license above, 


\title{
Behavioural Neurology
}

\section{Grey matter abnormalities are associated only with severe cognitive decline in early stages of Parkinson's disease}

\author{
Amée F. Wolters ${ }^{a, b, 1}$, Anja J.H. Moonen ${ }^{b, c, 1}$, Renaud Lopes ${ }^{d, e, f}$, \\ Albert F.G. Leentjens ${ }^{b, c, *}$, Annelien A. Duits ${ }^{b, c}$, Luc Defebure ${ }^{d, e, g}$, \\ Christine Delmaire ${ }^{d, e, f}$, Paul A. Hofman ${ }^{b, h}$, Frank C. van Bussel ${ }^{b, h}$ and \\ Kathy Dujardin ${ }^{d, e, g}$ \\ ${ }^{a}$ Department of Neurology, Maastricht University Medical Center, Maastricht, the Netherlands \\ ${ }^{\mathrm{b}}$ School for Mental Health and Neuroscience, Maastricht University Medical Center, Maastricht, the Netherlands \\ ${ }^{c}$ Department of Psychiatry and Neuropsychology, Maastricht University Medical Center, Maastricht, the \\ Netherlands \\ ${ }^{\mathrm{d}}$ Univ. Lille, U1171 - Degenerative \& Vascular Cognitive Disorders, Lille, France \\ e Inserm, U1171, Lille, France \\ ${ }^{\mathrm{f}} \mathrm{CHU}$ Lille, Neuroimaging Department, Lille, France \\ g CHU Lille, Neurology and Movement Disorders, Lille, France \\ ${ }^{\mathrm{h}}$ Department of Radiology, Maastricht University Medical Center, Maastricht, the Netherlands
}

\section{A R T I C L E I N F O}

\section{Article history:}

Received 24 September 2018

Reviewed 23 November 2018

Revised 11 June 2019

Accepted 26 September 2019

Action editor Brad Dickerson

Published online 24 October 2019

Keywords:

Cognition

Grey matter changes

Parkinson's disease

Dementia

\begin{abstract}
A B S T R A C T
Cognitive impairment is common in Parkinson's disease (PD), yet with large heterogeneity in the range and course of deficits. In a cross-sectional study, 124 PD patients underwent extensive clinical and neuropsychological assessment as well as a 3T MRI scan of the brain. Our aim was to identify differences in grey matter volume and thickness, as well as cortical folding, across different cognitive profiles as defined through a data-driven exploratory cluster analysis of neuropsychological data. The identified cognitive groups ranged from cognitively intact patients to patients with severe deficits in all cognitive domains, whilst showing comparable levels of motor disability and disease duration. Each group was compared to the cognitively intact PD group using voxel- and vertex-based morphometry. Results revealed widespread age-related grey matter abnormalities associated with progressive worsening of cognitive functions in mild PD. When adjusted for age, significant differences were only seen between cognitively intact and severely affected PD patients and these were restricted to the right posterior cingulate and the right precuneus. Reduced cortical thickness was seen in the right inferior temporal gyrus and reduced folding in the right temporal region. As these differences were not associated with age, we assume that they are associated with underlying pathology of the cognitive decline. Given the limited
\end{abstract}

* Corresponding author. Department of Psychiatry and Neuropsychology, Maastricht University Medical Center, P.O. Box 616, 6200 MD Maastricht, the Netherlands.

E-mail address: a.leentjens@maastrichtuniversity.nl (A.F.G. Leentjens).

1 These authors contributed equal to this paper and are duel first authors. 
involvement of grey matter differences, and the absence of differences in vascular changes across the groups, we hypothesize a more important role for white matter tract changes in cognitive decline in PD.

(c) 2019 Elsevier Ltd. All rights reserved.

\section{Introduction}

Cognitive deficits are common in Parkinson's disease (PD) and are increasingly recognized as an integral part of the disease. Up to $40 \%$ of PD patients report mild cognitive deficits already at the time of diagnosis (Yarnall et al., 2014). Approximately $30 \%$ of patients convert to dementia during the course of the disease (Svenningsson, Westman, Ballard, \& Aarsland, 2012), which increases up to $80 \%$ within 20 years after diagnosis (Aarsland, Andersen, Larsen, \& Lolk, 2003; Hely, Reid, Adena, Halliday, \& Morris, 2008). Yet, a substantial number of PD patients does not show any significant cognitive deficit, even years after being diagnosed (Foltynie, Brayne, Robbins, \& Barker, 2004; Janvin, Aarsland, Larsen, \& Hugdahl, 2003; Weintraub et al., 2011). Recent studies support the large heterogeneity in the range of cognitive deficits in PD, and several studies have attempted to identify distinctive cognitive profiles (Kehagia, Barker \& Robbins, 2010). However, many studies investigated cognitive dysfunction using the diagnostic categories as defined by the Movement Disorders Society (MDS) criteria published by Litvan et al. (2012). These include PD patients without cognitive impairment, patients with mild cognitive impairment (MCI), and patients with Parkinson's disease dementia (PDD). This approach does not allow the study of milder stages of cognitive decline, including cognitive deficits that do not reach the level for a diagnosis of MCI. In addition, although recent neuroimaging studies showed clear evidence for widespread cortical atrophy associated with MCI in PD and PD dementia (Burton, McKeith, Burn, Williams, \& O'Brien, 2004; Pereira et al., 2014; Song et al., 2011; Weintraub et al., 2011), using a classification approach may not only have masked neuroanatomical changes associated with more subtle cognitive deficits, but also obscure differences in possible sub-phenotypes that may correspond to different comorbid pathologies in more advanced stages of cognitive decline. This may be important, especially since a substantial number of PD patients appear to have neuropathological changes related to Alzheimer's disease in addition to diffuse Lewy body pathology (Jellinger, Seppi, Wenning, \& Poewe, 2002; Kalaitzakis \& Pearce, 2009; Kurosinski, Guggisberg, \& Götz, 2002).

In a prior study (Dujardin et al., 2013), we conducted a datadriven exploratory cluster analysis using retrospective neuropsychological test data from a large sample $(n=557)$ of PD patients. We identified five cognitive phenotypes, that were replicated and confirmed in a more recent study by performing a model-based confirmatory cluster analysis on prospective neuropsychological data from an independent sample of mild PD patients ( $n=156)$ (Dujardin et al., 2015). The five phenotypes represent different stages of cognitive decline ranging from cognitively intact patients to patients with severe dysfunction in all cognitive domains. In the present study, we aimed at identifying the neuroanatomical correlates of each of the identified cognitive phenotypes using structural MRI. More specifically, we were looking for regional differences in the rate of grey matter volume, cortical thickness, and cortical folding between the different groups. Considering the heterogeneity of the clinical presentation and course of cognitive deficits in $\mathrm{PD}$, we expect that different patterns of cerebral changes may underlie these different phenotypes. Detection of differences in grey matter may therefore increase our understanding of the underlying neurobiological changes that accompany the spectrum of cognitive disorders in PD. Moreover, if we find alterations in patients with only slight cognitive deficits, this exploratory approach could reveal a group at risk of further cognitive decline, who may be considered for early treatment options. Thirdly, studying grey matter alterations in PD may shed light on possible sub-phenotypes in more advanced stages of cognitive decline other than PD dementia, such as phenotypes with comorbid pathologies, such as Alzheimer's disease (AD).

We hypothesized that all groups would show a decline in grey matter volume, thickness and cortical folding compared with the cognitively intact group, with a progressive severity gradient. We further hypothesized to see profound hippocampal or medial temporal lobe grey matter deviations in those patients with more profound memory deficits, possibly reflecting a sub-phenotype of $\mathrm{PD}$ patients with comorbid $\mathrm{AD}$ pathology. We followed an explorative whole brain approach, with post hoc region-of-interest analyses.

\section{Materials and methods}

In this section we report how we determined our sample size, all data exclusions, all inclusion/exclusion criteria, whether inclusion/exclusion criteria were established prior to data analysis, all manipulations, and all measures used in the study. The study was performed accordance with the Declaration of Helsinki, and was approved by the local institutional review boards (METC azM/UM, NL42701.068.12; CPP Nord-Ouest IV, 2012-A 01317-36) ad registered in a clinical trial register (ClinicalTrials.gov Identifier: NCT01792843; https://clinicaltrials.gov/ct2/show/NCT01 792843?term $=$ NCT01792843\&rank=1). Legal and ethical restrictions prevent us from archiving our data in a public repository. The study data were pseudonymised and cannot be fully anonymised. In order to comply with the EU General Data Protection Regulation, the transfer of clinical data to an external research team would require the performance of regulatory proceedings by the principal investigator's institution. A new informed consent form would have to be signed (especially for requests outside the European Economic Area) and a new 
submission to the competent ethics committee would be necessary in order to authorise such a transfer. Data access requests should be directed to the corresponding author. Data will be released for research purposes subject to the completion of a data transfer agreement between our institution and the research institution requesting the data. This agreement will include the description of the requested variables, the purpose of the research, and the duties of both entities under personal data protection regulations.

\subsection{Participants}

One hundred fifty-six patients with idiopathic PD participated in this study (Dujardin et al., 2015). Patients were recruited from two independent European movement disorders clinics in Maastricht, the Netherlands $(n=75)$ and Lille, France $(n=81)$. The diagnosis of idiopathic PD was established by means of the United Kingdom Brain Bank diagnostic criteria for PD (Gibb \& Lees, 1988). Patients with moderate and severe dementia (defined as a score $>1$ at the Clinical Dementia Rating (Morris, 1993) scale and following the MDS criteria (Emre et al., 2007), patients older than 80 years and patients with neurodegenerative disorders other than PD were excluded from the study. Patients treated with deep brain stimulation or those meeting contra-indications for MRI were excluded likewise. All patients were using stable doses of antiparkinson medication for at least 2 months and were tested in their "on" state. All patients gave informed consent prior to participation in the study.

\subsection{Clinical assessment}

All patients underwent a thorough neuropsychiatric examination (see also [Dujardin et al., 2015]) consisting of the MDS Unified Parkinson's disease Rating Scale (MDS-UPDRS, sections I-IV) to measure severity and experiences of non-motor and motor symptoms, the Hoehn and Yahr staging scale to measure disease stage (Hoehn \& Yahr score), the 17-item Hamilton Depression Rating Scale (HAMD) to assess depressive symptoms, the Parkinson Anxiety Scale (PAS) to measure anxiety symptoms, the Lille Apathy Rating Scale (LARS) to measure levels of apathy, and the Lawton Instrumental Activities of Daily Living (IADL) to score activities of daily living (Goetz et al., 2008; Hamilton, 1960; Lawton \& Brody, 1969; Leentjens, Dujardin, Pontone, Starkstein, Weintraub \& Martinez-Martin, 2014; Sockeel, Dujardin, Devos, Deneve, Destée \& Defebvre, 2006). In addition, we checked for the presence of several medical conditions such as high blood pressure, hypercholesterolemia, diabetes, history of myocardial infarction, history of lower limb arteriopathy, cerebrovascular disease, sleep apnea, and symptoms of Rapid Eye Movement (REM) sleep behavior disorders. Antiparkinson medication was checked and doses were converted to levodopa equivalent daily dose (LEDD) according to the algorithm by Tomlinson et al. (2010).

\subsection{Neuropsychological assessment}

All patients also underwent an extensive neuropsychological test battery (see also [Dujardin et al., 2015]) measuring global cognition with the Mini Mental State Examination (MMSE) and the Mattis dementia rating scale (MDRS) as well as specific neuropsychological tests for multiple cognitive domains (Folstein, Folstein, McHughes, 1975; Mattis, 1976). Attention and working memory were assessed with the Digit span forward and backward and the Symbol Digit Modalities Test (SDMT) (Smith, 1982; Wechsler, 1986). Executive functioning was measured with the Trial Making Test (TMT, B/A ratio), the interference index of the Stroop word color test, and the word generation task (i.e., single and alternating phonemic conditions) (Reitan, 1992). Verbal episodic memory was measured by means of the Hopkins Verbal Learning Test (HVLT) (Brandt \& Benedict, 2001). We used the Boston Naming test and a categorical word generation task (i.e., animals) to assess language, and the short version of the Benton Judgment Line Orientation test (BJLO) to measure visuospatial functioning (Benton, Varney \& Hamsher, 1978; Graves, Bezeau, Fogarty \& Blair, 2004).

\subsection{Cognitive clusters}

Model-based confirmatory cluster analysis based on the neuropsychological test scores from all 156 patients revealed the following five-cluster model that was statistically superior and clinically the most relevant (see the supplementary material or [Dujardin et al., 2015] for a detailed description of the statistical procedure): 1) Cognitively intact patients with (above) average performance in all cognitive domains (25.6\% of the total group), 2) Cognitively intact patients with slight mental slowing and mild deficit in verbal fluency (i.e., SDMT and Semantic Fluency for animals) (26.9\%), 3) Patients showing mild cognitive deficits in attention, working memory, executive functioning, language and visuospatial functioning, as well as slight deficits in episodic verbal memory with intact recognition (37.2\%), 4) Patients with impaired overall cognitive efficiency and severe deficits in all cognitive domains, particularly executive functioning (3.2\%), and 5) Patients with impaired overall cognitive efficiency and severe deficits in all cognitive domains, particularly working memory and recall in verbal episodic memory (7.1\%). However, due to the small number of patients in cluster 4 and 5 , these groups were collapsed and considered as one cluster for further imaging analyses. Because the remaining four groups were not completely identical to the original clusters, we will use the term 'cognitive profile' rather than 'cluster'. The new groups, however, did not systematically differ from the original groups with respect to demographic, clinical and neuropsychological data.

\subsection{MRI data acquisition and analysis}

Patients were scanned at two sites (Maastricht and Lille) using the same 3T whole-body scanner (Achieva TX, Philips Healthcare, Best, the Netherlands) with identical software versions, MR sequences and an eight-channel SENSE head coil. Highresolution 3-dimensional T1-weighted images were acquired in the sagittal plane (TR/TE/TI 7.2/3.3/900 msec, $256 \times 256$ matrix, FOV $256 \times 240 \mathrm{~mm}^{2}, 1.0 \mathrm{~mm}^{3}$ isotropic voxel size after reconstruction), yielding 176 contiguous slices through the head.

\subsection{Optimized voxel-based morphometry}

Images were processed using the voxel-based morphometry (VBM) toolbox version 8 (VBM8; http://dbm.neuro.uni-jena.de/ $\mathrm{vbm} /$ ) within SPM8 (Welcome Trust Centre for Neuroimaging, 
London, UK; http://fil.ion.ucl.ac.uk/spm/software/spm8/) in MATLAB 7.14 (Math-works Inc., Natick, MA, USA). Default settings were used unless otherwise specified. Prior to image analyses, all images were screened for artifacts and large anatomical abnormalities, such as ischemic strokes. Subjects displaying ischemic strokes or significant artifacts were excluded from our analysis. The Fazekas scale was applied to quantify the amount of white matter lesions related to chronic small vessel ischemia, on the MRI images (Fazekas, Chawluk, Alavi, Hurting, \& Zimmerman, 1987). Afterwards, images were and manually re-orientated in SPM8, such that they were centered on the anterior commissure. For spatial normalization, we applied the high-dimensional Dartel normalization approach (VBM-Dartel; Ashburner et al., 2007). The next step involved modulated normalization followed by segmentation of images into grey matter, white matter and cerebrospinal fluid, in order to obtain regional grey matter values and account for individual differences in global brain size. After checking the homogeneity of the sample, the normalized modulated images were smoothed with a standard Gaussian kernel of $8 \mathrm{~mm}$, full width at half maximum (FWHM).

\subsection{Vertex-based morphometry}

Structural T1 images were processed using the Freesurfer software version 5.3 (http://surfer.nmr.mgh.harvard.edu/, version 5.3). This included the preprocessing steps of non-uniform signal correction, signal and spatial normalizations, skull stripping and brain tissue segmentation (Dale, Fischl, \& Sereno, 1999). Triangulated surface models of the inner and outer cortical surfaces were obtained for each patient. Surface reconstruction was reviewed by an expert and corrected with minor manual intervention when appropriate. After inflation and parameterization, cortical surface models were registered to a common surface template (Freesurfer's fsaverage) using a multiscale nonrigid spherical registration procedure minimizing folding pattern differences across individuals (Fischl, 1999).

The cortical thickness was obtained by reconstructing representations of inner and outer surfaces and then calculating the distance between those surfaces using the t-link method, defined as the Euclidean distance between linked vertices on inner and outer surfaces (Fischl, 2012; Lerch \& Evans, 2005). Cortical thickness maps were finally smoothed with a 20-mm FWHM Gaussian surface kernel.

The local gyrification index was measured on each vertex from the outer cortical surface. This index is a measurement of the degree of cortical folding that quantifies the amount of cortex buried within the sulcal folds in the surrounding circular region. A 3D approach was used to take into account the 3D nature of the cortical folds and with the ability to localize abnormalities in cortical folding. The local gyrification index ratio between the outer surface and outer hull at the centre of a $20-\mathrm{mm}$ spherical region was computed to each vertex (Schaer et al., 2008).

\subsection{Statistical analysis}

The smoothed, modulated, normalized grey matter VBM data were analyzed in SPM8. T-tests were conducted to identify differences in whole brain grey matter volume between the patient groups. An absolute threshold of .1 was used, by which only voxels with grey matter values $>.1$ were counted. Correction for global volume change was not necessary, since we already applied this correction directly to the data as part of the (nonlinear) modulation step (recommended by the VBM8 manual). We ran the analysis twice, once with center, gender and education entered as covariates into the design matrix and once including age as an additional covariate in order to make the age related effects on grey matter volume differences more explicit.

An exploratory whole brain analysis was performed with a threshold for statistical significance of $p<.001$ without correction for multiple comparisons. Clusters were considered significant using the family-wise error (FWE) threshold of $p_{\text {FWE }}<.05$, corrected for multiple comparisons based on the random field theory. In addition, post-hoc regions of interest analyses were performed using mask images for these regions from the SPM8 Anatomy Toolbox. Significant group differences are reported at $p_{\mathrm{sCV}}<.05$ (small volume correction, i.e., family wise error correction within the search volume of $8 \mathrm{~mm}$ ). The $x, y$, and $z$ coordinates of areas displaying significant whole brain or regions of interest group differences in grey matter volume were identified using the Brede Database (Nielsen, 2003), the Nonlinear Yale MNI to Talairach Conversion Algorithm (Lacadie, Fulbright, Rajeevan, Constable, \& Papademetris, 2008), and the SPM Anatomy Toolbox.

Between-group differences in cortical thickness and cortical folding were investigated with the same approach as the VBM approach adapted for surface-based analysis. Statistical analyses were performed using Keith Worsley's SurfStat toolbox (http://www.math.mcgill.ca/keith/surfstat/). Ttests were performed to investigate between-group differences with center, gender and education level as covariates. Significant vertices were firstly obtained with a threshold of $p$ $<.001$ without correction for multiple comparisons. Clusters were secondly considered significant using FWE threshold of $p_{F W E}<.05$, corrected for multiple comparisons based on the random field theory. We repeated the analysis including age as an additional covariate in order to assess age related effects on cortical thickness and cortical folding.

Statistical analysis of the behavioral data was performed using the Statistical Package for Social Sciences version 22 (SPSS, Chicago). The numerical variables were described as means and standard deviations and the categorical variables as medians, frequencies and percentages. Group comparisons were performed for demographical and clinical variables, as well as for the cognitive variables, using an analysis of variance with generalized linear model with Tukey correction for multiple testing when data distribution was normal, and a Kruskal-Wallis test was used for non-normal distributions. Categorical variables were compared with Chi-square test. A $p$ value $<.05$ was considered significant. No part of the study analyses was pre-registered prior to the research being conducted.

\section{Results}

\subsection{Demographics}

Of the 156 included patients, 133 patients had an MRI scan. An extensive data quality check resulted in the exclusion of 9 
patients (6 due to large motion artifacts and 3 due to large lesions). Table 1 shows the demographic and clinical characteristics of the patients over the 4 cognitive profiles. Patients in group 1 were significantly younger than those in the three other groups and received more years of formal education than patients in group 3 and 4 . Patients from group 4 showed more disease-related speech abnormalities (i.e., hypophonia) than those in the other three groups and more frequent and more severe hallucinations than patients in group 1 and 2 . Mild dementia (based on clinical interpretation of test scores and level of autonomy) was more frequent in group 4 than in the other groups. Group 3 showed more symptoms of persistent and avoidance anxiety and more symptoms of apathy than group 1.

\subsection{Neuropsychological data}

Table 2 shows the neuropsychological test data of the four patient groups per cognitive domain. Patients from group 1 and 2 were cognitively intact, with average to high levels of performance in all cognitive domains in group 1, and only slight slowing of information processing speed and verbal fluency in group 2 (i.e., SDMT and Semantic Fluency for animals, respectively). Group 3 scored lower on global cognition (i.e., MMSE and MDRS) than the cognitively intact groups, yet still within the normal range. Patients in group 3 further scored lower on tests measuring attention, working memory, episodic verbal memory (except recognition), executive functions, language and visuospatial functions. Patients in group 4 showed impaired global cognition and severe deficits in all cognitive domains.

\subsection{Grey matter volume}

A first analysis, using center, gender and education level as covariates showed significant reduced grey matter volume between several groups in a number of brain regions, located both in the temporal, parietal and frontal lobe areas (See supplementary Table 1 for specific information regarding the location and MNI coordinates of these regions). However, after including age as a covariate into the model as well, no differences in grey matter volume

Table 1 - Demographic and clinical features of the four patient groups.

\begin{tabular}{|c|c|c|c|c|c|c|c|c|c|c|}
\hline \multirow[t]{2}{*}{ Group (n) } & \multicolumn{2}{|c|}{ Group 1 (34) } & \multicolumn{2}{|c|}{ Group 2 (33) } & \multicolumn{2}{|c|}{ Group 3 (44) } & \multicolumn{2}{|c|}{ Group 4 (13) } & \multirow[t]{2}{*}{$p$ values } & \multirow[t]{2}{*}{ Post-hoc tests } \\
\hline & Mean & SD & Mean & SD & Mean & SD & Mean & SD & & \\
\hline \multicolumn{11}{|l|}{ Demographic } \\
\hline Sex (\% male) & 70.6 & & 75.8 & & 61.4 & & 69.2 & & .5897 & \\
\hline Age & 60.0 & 8.4 & 66.1 & 5.6 & 66.9 & 8.0 & 71.29 & 5.88 & $<.0001$ & $1<2,3,4$ \\
\hline Formal education (y) & 13.8 & 3.2 & 13.5 & 4.2 & 11.5 & 3.5 & 9.1 & 2.1 & $<.0001$ & $1>3,4 ; 2>3,4 ; 3>4$ \\
\hline \multicolumn{11}{|l|}{ Clinical } \\
\hline Disease duration (y) & 8.1 & 5.2 & 8.9 & 7.4 & 8.8 & 4.9 & 9.4 & 4.2 & .8909 & NA \\
\hline MDS_UPDRS3 score & 25.5 & 10.4 & 30.2 & 12.0 & 28.1 & 11.2 & 31.9 & 16.7 & .2741 & NA \\
\hline Hoehn \& Yahr stage & 1.9 & .4 & 2.1 & .6 & 2.2 & .6 & 2.3 & .5 & .0405 & \\
\hline Dyskinesia (\%) & 17.6 & & 15.2 & & 27.3 & & 30.8 & & .4595 & \\
\hline Postural instability (score MDS_UPDRS3_12) & .4 & .6 & .6 & .7 & .7 & .9 & .9 & 1.1 & .2617 & NA \\
\hline Hypophonia (score MDS_UPDRS3_1) & .9 & 6 & .9 & .7 & 1.0 & .7 & 1.6 & .9 & .0067 & $4<1,2,3$ \\
\hline REM sleep behavior disorder (\%) & 23.5 & & 36.4 & & 45.5 & & 38.5 & & .2594 & NA \\
\hline IADL (\% with full autonomy) & 76.5 & & 66.7 & & 34.1 & & .0 & & $<.0001$ & NA \\
\hline Dementia (\%) & .0 & & .0 & & 11.4 & & 46.2 & & $<.0001$ & NA \\
\hline \multicolumn{11}{|l|}{ Medication } \\
\hline LEDD (mg/day) & 721.3 & 675.1 & 732.6 & 487.3 & 874.94 & 543.6 & 861.6 & 285.4 & .5433 & NA \\
\hline Levodopa (\%) & 82.4 & & 84.8 & & 90.9 & & 100.0 & & .3290 & NA \\
\hline Dopamine agonist (\%) & 58.8 & & 60.6 & & 47.7 & & 69.2 & & .4689 & NA \\
\hline Antidepressant (\%) & 11.8 & & 12.1 & & 18.2 & & 15.4 & & .8388 & NA \\
\hline Benzodiazepine (\%) & 2.9 & & 6.1 & & 15.9 & & 15.4 & & .1953 & NA \\
\hline \multicolumn{11}{|l|}{ Neuropsychiatry } \\
\hline Hamilton depression rating scale & 6.0 & 4.4 & 5.2 & 5.5 & 6.4 & 4.2 & 5.4 & 4.5 & .7369 & NA \\
\hline Lille apathy rating scale & -27.2 & 6.1 & -26.8 & 6.3 & -23.1 & 6.9 & -22.5 & 7.3 & .0096 & $1<3$ \\
\hline PAS (score of persistent anxiety) & 3.4 & 3.9 & 4.2 & 4.2 & 6.4 & 4.9 & 6.3 & 5.5 & .0181 & $1<3$ \\
\hline PAS (score of episodic anxiety) & .7 & 1.1 & 1.1 & 2.0 & 1.1 & 1.8 & 1.1 & 1.6 & .7286 & NA \\
\hline PAS (score of avoidance) & .5 & 1.5 & 1.0 & 2.0 & 1.6 & 1.7 & 1.2 & 1.3 & .0457 & $1<3$ \\
\hline Hallucinations (\%) & 5.9 & & 6.1 & & 18.2 & & 30.8 & & .0538 & NA \\
\hline \multicolumn{11}{|l|}{ Vascular risk factors } \\
\hline High blood pressure (\%) & 20.6 & & 27.3 & & 22.7 & & 38.5 & & .6103 & NA \\
\hline Hypercholesterolemia (\%) & 17.6 & & 24.2 & & 25.0 & & 15.4 & & .7920 & NA \\
\hline Diabetes (\%) & 2.9 & & 12.1 & & 9.1 & & 7.7 & & .5722 & NA \\
\hline Sleep apnea syndrome (\%) & 11.8 & & 18.2 & & 18.2 & & 15.4 & & .8676 & NA \\
\hline History of heart infarct (\%) & .0 & & 6.1 & & 4.5 & & .0 & & .4468 & NA \\
\hline Lower limb arteriopathy (\%) & 8.8 & & 12.1 & & .0 & & .0 & & .0838 & NA \\
\hline
\end{tabular}

MDS_UPDRS3 = Movement Disorders Society Unified Parkinson's Disease Rating Scale-Part III (severity of motor symptoms); IADL = instrumental activities of daily living; LEDD = Levodopa Equivalent Daily Dose; PAS=Parkinson Anxiety Scale; NA = Not applicable. Due to low frequency of observations, we only mentioned qualitative results of overall group comparisons. 
Table 2 - Cognitive test performance (mean and standard deviation) of the four patient groups.

\begin{tabular}{|c|c|c|c|c|c|c|c|c|c|c|}
\hline & \multicolumn{2}{|c|}{ Group 1} & \multicolumn{2}{|c|}{ Group 2} & \multicolumn{2}{|c|}{ Group 3} & \multicolumn{2}{|c|}{ Group 4} & \multirow[t]{2}{*}{$P$ values } & \multirow[t]{2}{*}{ Post-hoc tests } \\
\hline & Mean & SD & Mean & SD & Mean & SD & Mean & SD & & \\
\hline $\begin{array}{l}\text { Number of subjects (\%) } \\
\text { Global cognition }\end{array}$ & \multicolumn{2}{|c|}{$34(27.4)$} & $33(26.6)$ & & \multicolumn{2}{|l|}{$44(35.5)$} & \multicolumn{2}{|l|}{$13(10.5)$} & & \\
\hline MMSE score (/30) & 28.9 & 1.1 & 28.5 & 1.6 & 27.1 & 2.3 & 24.6 & 3.5 & $<.0001$ & $1>3,4 ; 2>4 ; 3>4$ \\
\hline $\begin{array}{l}\text { MDRS score (/144) } \\
\text { Attention and working memory }\end{array}$ & 140.9 & 3.11 & 139.9 & 3.3 & 134.6 & 5.55 & 125.5 & 9.1 & $<.0001$ & $1>3,4 ; 2>3,4 ; 3>4$ \\
\hline WAIS-R forward digit (/14) & 8.8 & 1.7 & 7.8 & 1.9 & 6.8 & 2.5 & 6.9 & 2.5 & .0004 & $1>3,4$ \\
\hline WAIS-R backward digit (/14) & 6.7 & 1.5 & 6.3 & 1.5 & 4.7 & 1.6 & 3.9 & 1.4 & $<.0001$ & $1>3,4 ; 2>3,4$ \\
\hline $\begin{array}{l}\text { SDMT: number in } 90 \mathrm{sec} \\
\text { Executive functions }\end{array}$ & 55.7 & 7.0 & 43.5 & 3.4 & 33.2 & 6.7 & 18.1 & 9.2 & $<.0001$ & $1>2,3,4 ; 2>3,4 ; 3>4$ \\
\hline Trail Making Test (time B/time A) & 2.2 & .6 & 2.5 & .7 & 2.8 & .8 & 3.0 & 1.1 & .0005 & $1>3,4$ \\
\hline Stroop: interference index & 1.6 & .4 & 1.7 & .2 & 2.1 & .6 & 2.4 & 1.0 & $<.0001$ & $1>3,4 ; 2>3,4$ \\
\hline Stroop: errors & .5 & 1.9 & .9 & 1.2 & 4.1 & 4.3 & 18.9 & 15.0 & $<.0001$ & $1>3,4 ; 2>4 ; 3>4$ \\
\hline Phonemic fluency: naming in $60 \mathrm{sec}$ & 15.4 & 5.3 & 14.9 & 2.9 & 10.9 & 3.9 & 7.5 & 3.2 & $<.0001$ & $1>3,4 ; 2>3,4 ; 3>4$ \\
\hline $\begin{array}{l}\text { Alternating fluency: naming in } 60 \mathrm{sec} \\
\text { Episodic memory }\end{array}$ & 14.7 & 4.1 & 13.1 & 3.6 & 8.5 & 3.5 & 6.3 & 3.3 & $<.0001$ & $1>3,4 ; 2>3,4$ \\
\hline HVLT Learn trial 1 (/12) & 7.4 & 1.7 & 6.9 & 1.5 & 5.5 & 2.1 & 3.7 & 1.5 & $<.0001$ & $1>3,4 ; 2>3,4 ; 3>4$ \\
\hline HVLT Learn total (/36) & 28.3 & 4.0 & 27.1 & 3.2 & 23.3 & 4.1 & 16.9 & 4.6 & $<.0001$ & $1>3,4 ; 2>3,4 ; 3>4$ \\
\hline HVLT delayed recall (/12) & 10.0 & 1.7 & 9.9 & 1.8 & 8.0 & 2.3 & 4.6 & 3.0 & $<.0001$ & $1>3,4 ; 2>3,4 ; 3>4$ \\
\hline HVLT recognition hits (/12) & 11.6 & .7 & 11.6 & .8 & 11.1 & 1.1 & 9.6 & 2.1 & .0004 & $1>4 ; 2>4 ; 3>4$ \\
\hline $\begin{array}{l}\text { HVLT number of intrusions } \\
\text { Language }\end{array}$ & .5 & 1.0 & 1.1 & 1.8 & 1.5 & 2.3 & 2.2 & 1.4 & .0018 & $1>4$ \\
\hline Boston naming test (/15) & 13.9 & 1.2 & 13.4 & 1.8 & 11.2 & 2.6 & 10.9 & 3.0 & $<.0001$ & $1>3,4 ; 2>3,4$ \\
\hline $\begin{array}{l}\text { Animal naming in } 60 \mathrm{sec} \\
\text { Visuospatial functions }\end{array}$ & 25.7 & 4.9 & 19.8 & 3.4 & 15.1 & 4.0 & 10.7 & 5.5 & $<.0001$ & $1>2,3,4 ; 2>3,4 ; 3>4$ \\
\hline Judgment of line orientation & 12.7 & 2.0 & 12.5 & 3.4 & 10.7 & 2.9 & 7.9 & 2.7 & $<.0001$ & $1>3,4 ; 2>3,4 ; 3>4$ \\
\hline
\end{tabular}

between any of the groups remained significant. When applying a more liberal statistical threshold of $p<.005$ reduced grey matter volume was revealed in group 4 compared to group 1 in the left medial temporal pole (BA 38 ), and a trend within the right posterior cingulate gyrus (BA 31) and right precuneus (BA 7) ( $\left.p_{F W E}=.052\right)$. Fig. 1 demonstrates the areas as identified by the VBM analyses with correction for center, gender, age and level of education where group 4 showed differences in grey matter volume compared with group 1 . See also Table 3 for specific details about location and MNI coordinates.

\subsection{Cortical thickness and cortical folding}

Fig. 2 shows the statistical comparisons for which cortical thickness were significantly reduced between two groups using center, gender, age and education level as covariates. Without correction for age, a significantly reduced cortical thickness between groups was observed in several brain regions, most dominantly in the temporal regions. However, after including age as a covariate into the model, only reduced cortical thickness of group 4 compared with group 1 was observed in the right inferior temporal gyrus (Fig. 2).

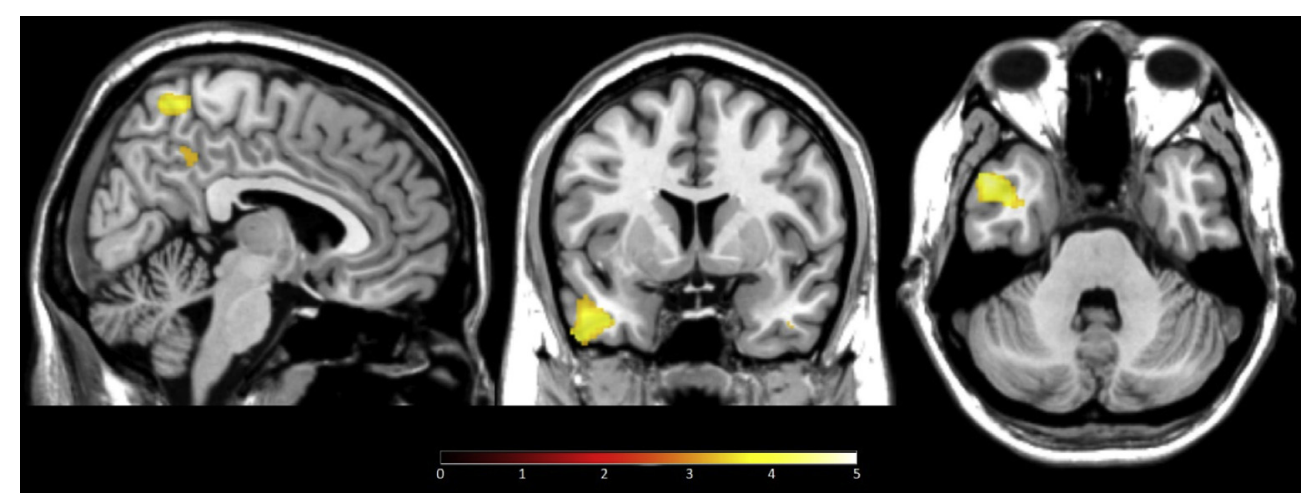

Fig. 1 - VBM group comparisons of grey matter volume with centre, gender, education level and age as covariates. The threshold was set at $p_{\text {FWE }}<.05$, corrected for multiple comparisons based on the random field theory. A decreased grey matter volume was found in the left medial temporal pole in groups 4 versus group 1, and a trend within the right posterior cingulate gyrus and right precuneus. The color scale represents the T-value corresponding to the significant cluster. 
Table 3 - Location and MNI coordinates of clusters of significant grey matter volume loss after comparing all groups to the cognitively intact PD group (group 1).

\begin{tabular}{|c|c|c|c|c|c|c|c|}
\hline \multirow[t]{2}{*}{ Comparison } & \multirow[t]{2}{*}{ Location } & \multirow[t]{2}{*}{ Cluster size $(k)$} & \multicolumn{3}{|c|}{ MNI coordinates } & \multirow[t]{2}{*}{ T-value } & \multirow[t]{2}{*}{$p$-value } \\
\hline & & & $x$ & $\mathrm{y}$ & $z$ & & \\
\hline \multirow[t]{6}{*}{ Group $1>4$} & L medial temporal pole 38 & 1753 & -48 & 12 & -35 & 4.57 & .044 \\
\hline & L medial temporal pole 38 & & -40 & 3 & -33 & 4.23 & \\
\hline & L medial temporal pole 38 & & -54 & 6 & -41 & 4.04 & \\
\hline & R precuneus 7 & 1829 & 8 & -54 & 64 & 4.24 & .052 \\
\hline & $\mathrm{R}$ posterior cingulate gyrus 31 & & 14 & -55 & 33 & 3.66 & \\
\hline & $\mathrm{R}$ posterior cingulate gyrus 31 & & 16 & -43 & 37 & 3.57 & \\
\hline
\end{tabular}

Cluster size denotes the extent of the activation cluster by number of significant voxels $(k)$. MNI coordinates refer to the location of the maximally activated voxel (peak) within an activation cluster. Results are considered significant at $P_{\mathrm{FWE}}<.05$ (FWE-corrected based on random field theory), corrected for center, gender, age and education. Numbers refer to Brodmann areas (location). L = left; $\mathrm{R}=$ right.

See Table 4 for specific details about location and MNI coordinates of the significant age-adjusted results.

After including age, center, gender and education level as a covariate, the cortical folding was solely statistically reduced in group 4 compared with group 1. Group 4 showed less local gyrification index values in right temporal regions (Fig. 2). See Table 4 for specific details about location and MNI coordinates.

(For the results of the cortical thickness and cortical folding analysis corrected for center, gender and education level, but not for a age, we refer to the supplementary Table 2).

\subsection{Small vessel disease}

The amount of white matter lesions related to chronic small vessel disease, was scored on all MRI images according to the Fazekas scale. All groups displayed a median Fazekas score of 1. Analysis using a Chi-Square test did not reveal a significant difference between groups $(P=.327)$.

\section{Discussion}

The age-corrected results of this study show a significant difference in grey matter volume only between the group with severe cognitive decline (group 4) and the cognitively intact group (group 1). More specifically, group 4 showed a reduced grey matter volume within the right posterior cingulate gyrus (BA 31) and right precuneus (BA 7). Furthermore, our results gave no indication for a regional progressive severity gradient of reduced grey matter volume associated with more severe cognitive decline. In other words, we found no difference in the degree of grey matter volume in specific brain regions between patients with slight versus more severe cognitive deficits. Instead, we observed pathological changes in patients with more severe cognitive decline (group 4) compared to cognitively intact patients (group 1). In addition, a significant reduction in cortical thickness and cortical folding between group 4 and group 1 was predominantly seen in the right temporal regions. No significant results in cortical folding or

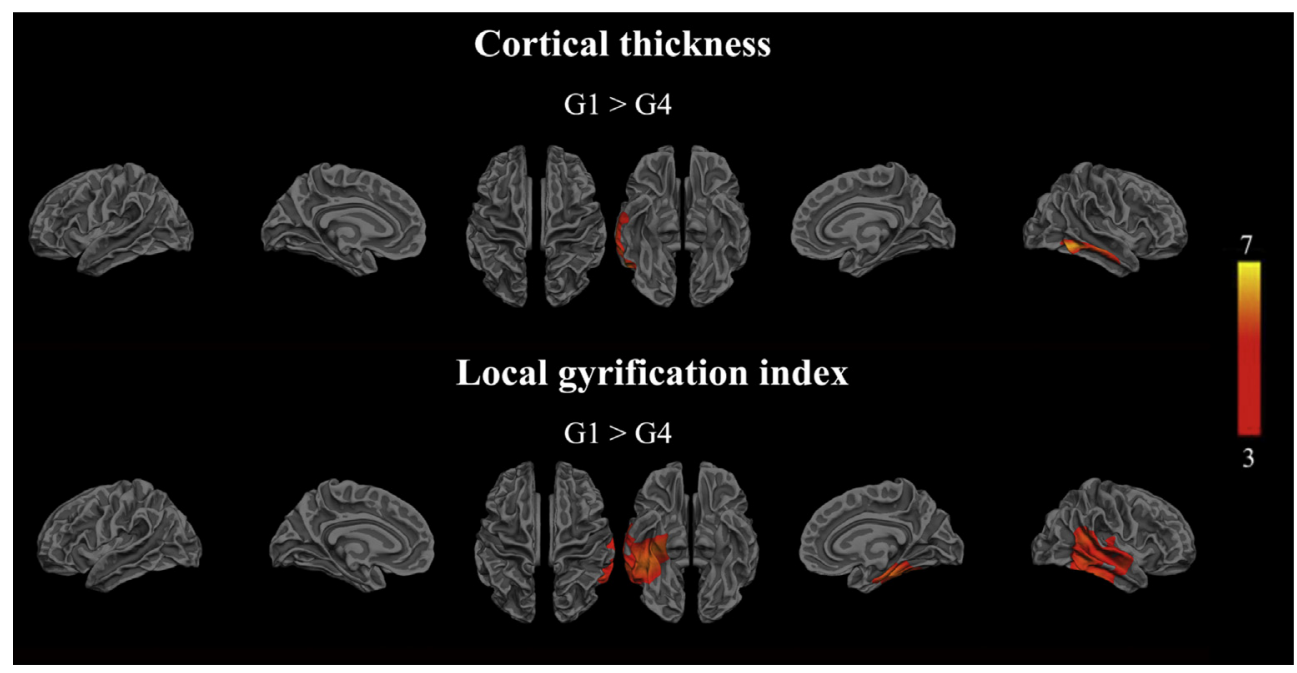

Fig. 2 - Between-group comparisons of cortical thickness and cortical folding values with center, gender, education level and age as covariates. The threshold was set at $p_{\mathrm{FWE}}<.05$, corrected for multiple comparisons based on the random field theory. The color scale represents the T-value corresponding to the significant vertices. 
Table 4 - Significant differences in cortical thickness and local gyrification index between patient groups.

\begin{tabular}{|c|c|c|c|c|c|c|c|}
\hline \multirow[t]{2}{*}{ Comparison } & \multirow[t]{2}{*}{ Location } & \multirow[t]{2}{*}{ Cluster size (\# of vertices) } & \multicolumn{3}{|c|}{ MNI coordinates } & \multirow[t]{2}{*}{ T-value } & \multirow[t]{2}{*}{$p$-value } \\
\hline & & & $x$ & $\mathrm{y}$ & $\mathrm{z}$ & & \\
\hline \multicolumn{8}{|c|}{ Cortical thickness } \\
\hline \multirow[t]{2}{*}{ Group $1>4$} & $\mathrm{R}$ inferior temporal gyrus & 2616 & 58 & -54 & -11 & 5.92 & .016 \\
\hline & $\mathrm{R}$ middle temporal gyrus & & 67 & -21 & -11 & 4.64 & \\
\hline \multicolumn{8}{|c|}{ Local gyrification index } \\
\hline \multirow[t]{5}{*}{ Group $1>4$} & $\mathrm{R}$ medial occipitotemporal sulcus & 18749 & 36 & -25 & -23 & 5.21 & $3.10^{-4}$ \\
\hline & $\mathrm{R}$ lateral occipitotemporal sulcus & & 45 & -42 & -19 & 4.73 & \\
\hline & R superior temporal sulcus & & 51 & -45 & 15 & 4.46 & \\
\hline & $\mathrm{R}$ inferior temporal sulcus & & 58 & -47 & -9 & 4.46 & \\
\hline & $\mathrm{R}$ circular insula sulcus & & 38 & -24 & 5 & 3.85 & \\
\hline
\end{tabular}

Cluster size denotes the extent of the activation cluster by number of significant voxels $(k)$. MNI coordinates refer to the location of the maximally activated vertex (peak) within an activation cluster. Results are considered significant at $P_{\mathrm{FWE}}<.05$ (FWE-corrected based on random field theory), corrected for center, gender, age and education. $\mathrm{L}=$ left; $\mathrm{R}=$ right.

cortical thickness were observed between the other groups when age was introduced as a covariate. Moreover, we did not find any significant differences in Fazekas scores between the groups.

Patients in all four groups were at relatively early motor stages, showing no significant differences in severity of motor disability, disease duration or medication use. Interestingly, patients in group 3 (comparable to 'PD-MCI') displayed significantly more severe symptoms of anxiety and apathy when compared to the cognitively intact groups. Therefore, this data-driven approach indicates that it is useful to also consider non-motor symptoms such as neuropsychiatric symptoms when classifying PD patients, as this probably provides a more representative classification.

The fourth group, characterized by global and severe cognitive decline in multiple cognitive domains (e.g., verbal episodic memory and visuospatial deficits), showed reduced cortical thickness and cortical folding in the medial temporal lobe. The involvement of medial temporal areas is in line with other VBM studies (Burton et al., 2004; Weintraub et al., 2011) and possibly reflects more widespread Lewy body pathology as well as a marker for conversion to PDD. Within group 4, six patients already met the diagnostic criteria for mild PDD, while the remaining patients $(n=7)$ could be classified as multiple domains MCI (Litvan et al., 2012). Another characteristic of this group was the high rate of visual hallucinations. The association between visual hallucinations and severe cognitive decline (Aarsland et al., 2004), as well as a higher risk for developing dementia (Aarsland et al., 2003), has been related to both Lewy body pathology in the temporal lobe (Harding, Broe, \& Halliday, 2002) and to cholinergic deficits (Perry et al., 1990). Moreover, group 4 was the only group with a higher score of hypophonia, an axial sign which has also been associated with cognitive worsening (Uc et al., 2009).

Interestingly, autopsy studies revealed that a substantial number of $\mathrm{PD}$ patients appear to have neuropathological changes related to $\mathrm{AD}$ in addition to diffuse Lewy body pathology (Jellinger et al., 2002; Kalaitzakis \& Pearce, 2009; Kurosinski et al., 2002). As such, more advanced stages of cognitive decline in PD may reflect an interaction of different neurodegenerative processes such as comorbid AD-related neurodegenerative changes (Sabbagh et al., 2009). Moreover, the combination of amyloid- and tau-related pathological changes and limbic and cortical Lewy body abnormalities appear to be the strongest correlate of dementia in PD (Compta et al., 2011). Other factors, such as neuroinflammation, synaptic pathological changes and neurotransmitters may be involved as well. In our sample, we did not find evidence for a contribution of vascular changes to cognitive decline.

Contrary to our expectations, grey matter changes were not widespread and only visible between the cognitive intact group and the group with the most severe cognitive decline. This may indicate that grey matter alternations may not be as important as previously thought, at least in the early stages of cognitive decline. Since we also did not find differences in vascular lesions between the groups, this may point in the direction that alterations in white matter tracts may play a role. This hypothesis is supported by results of previous studies, which investigated both white and grey matter alterations in a group of PD patients. These authors conclude that changes in white matter precede grey matter alterations (Duncan et al., 2016; Rektor et al., 2018). Grey matter atrophy is an expression of neuronal cell death, a phenomenon that is seen in relatively late stages of PD dementia. In contrast to this, the earliest stages of cognitive impairment in PD are characterized by axonal and synaptic changes. Other MRI techniques, such as diffusion-weighted imaging, are able to detect alterations in white matter integrity related to axonal damage and might therefore be more useful in early stages of cognitive decline in PD (Weil, Costantini, \& Schrag, 2018).

Levy (2007) proposed a model for the relationship between PD and advanced aging, stating that advanced age is associated with a faster rate of motor progression, decreased levodopa responsiveness, more severe gait and postural impairments, reduced autonomy, more severe cognitive impairment and a higher risk for developing dementia in PD. The model further suggests a substantial role for aging in the pathogenesis of $\mathrm{PD}$, by means of a biological interaction between the effects of the disease process and aging in nondopaminergic structures, which may indeed denote the involvement of monoaminergic and cholinergic structures in more advanced stages of PD. As such, it is not surprising that 
most of the between group differences in our study were no longer significant after the correction for age.

For this study two different methods to assess grey matter alterations were combined, VBM and surface-based cortical thickness approaches. We believe that both of the methods add complementary information. VBM is able to evident grey matter differences based on voxel values on the whole brain, while surface-based cortical thickness approaches are limited to the cortex and provide more subtle information about cortical thickness (Clarkson et al., 2011). Furthermore, VBM quantifies grey matter volume changes between groups, which is a combination of information about the cortical thickness and surface areas. Several previous studies showed the additional value of combining these methods, both in healthy subjects (Hutton, Draganski, Ashburner, \& Weiskopf, 2009) and PD patients (Gerrits et al., 2016).

Our study has several limitations. First, due the research question and study design (data-driven approach in order to identify cognitive phenotypes in PD), we did not include a healthy control group. The cognitively intact PD patients (group 1) was considered our reference group. Consequently, we were not able to identify (early) differences in grey matter volume loss between this group and healthy controls. Also, we were not able to disentangle the absolute contribution of disease to cognitive decline independently of age. Age appeared to be an overpowering factor in the model, possibly masking other factors. Secondly, the number of patients in the original clusters 4 and 5 was low, because of the exclusion of patients with moderate or severe dementia to limit imaging artifacts and missing data due to misunderstanding of instructions. However, despite collapsing these two clusters, the remaining group 4 still remained very small $(n=13)$. Results for this group after correction for age would probably have reached the level of significance without a more liberal threshold if the sample had been larger. Thirdly, patients were recruited from tertiary referral centers, making the sample possibly not representative for the general population. Fourthly, spatial normalization and image segmentation in VBM is fully automated, which may lead to difficulties in detecting subtle changes in areas that show high variability among elderly and diseased groups (e.g., the hippocampus) and may lead to misclassification of voxels due to reduced tissue contrast. However, the use of a strict threshold for significance reduced the possibility for such errors substantially. Moreover, the use of automated whole brain analysis methods for evaluating brain structure in diseased brains is less prone to subjectivity associated with regions-of-interest-based methods and is not restricted by a priori assumptions about specific regions of interest. Another important aspect concerns the use of a cross-sectional design, which limited us to merely describe the clinical characteristics and neural substrates of the separate cognitive profiles. A follow-up of the identified groups would be essential to explore differences in disease progression. Moreover, this may reveal the extent to which neural substrates associated with early markers of cognitive impairment (e.g., slowed mental speed) can be used as a predictor of severe cognitive decline and conceivable conversion to dementia. An important strength of our study is the use of a data-driven approach. Groups were not previously defined based on diagnostic criteria for MCI and PDD. This allowed recognition of different phenotypes regarding cognitive functioning and other non-motor symptoms in early PD patients with similar motor disability. We believe that a data-driven approach to define groups can be very useful for identifying early markers of cognitive decline in PD.

In conclusion, our results revealed grey matter abnormalities associated only with severe cognitive decline in PD. Reduction of cortical volume occurs in the right posterior cingulate and precuneus, while reduced cortical thickness and folding occurs bilateral in the medial temporal regions. These seem to be attributable to processes of cognitive decline and not associated with age. The absence of cortical changes and vascular lesions point in the direction of a more prominent involvement of white matter tract alterations. Future studies should preferably be prospective and include a larger number of PD patients with more advanced cognitive decline. Studies may also benefit from more specific interest in white matter changes.

\section{Open practices}

The study in this article earned a Preregistered badge for transparent practices.

\section{Funding}

This study was funded by the Michael J. Fox Foundation for Parkinson's Research.

\section{Competing interests}

The authors have no conflicts of interest to declare.

\section{CRediT authorship contribution statement}

Amée F. Wolters: Formal analysis, Writing - original draft. Anja J.H. Moonen: Data curation, Formal analysis, Writing - original draft. Renaud Lopes: Formal analysis, Writing - review \& editing. Albert F.G. Leentjens: Conceptualization, Supervision, Writing review \& editing. Annelien A. Duits: Supervision, Writing - review \& editing. Luc Defebvre: Writing - review \& editing. Christine Delmaire: Writing - review \& editing. Paul A. Hofman: Writing - review \& editing. Frank C. van Bussel: Writing - review \& editing. Kathy Dujardin: Conceptualization, Funding acquisition, Supervision, Writing - review \& editing.

\section{Supplementary data}

Supplementary data to this article can be found online at https://doi.org/10.1016/j.cortex.2019.09.015.

\section{R E F E R E N C E S}

Aarsland, D., Andersen, K., Larsen, J. P., \& Lolk, A. (2003). Prevalence and characteristics of dementia in Parkinson disease: An 8-year prospective study. Archives of Neurology, 60, 387-392. 
Aarsland, D., Andersen, K., Larsen, J. P., Perry, R., WentzelLarsen, T., Lolk, A., et al. (2004). The rate of cognitive decline in Parkinson disease. Archives of Neurology, 61, 1906-1911.

Ashburner, J. (2007). A fast diffeomorphic image registration algorithm. NeuroImage, 38, 95-113.

Benton, A. L., Varney, N. R., \& Hamsher, K. S. (1978). Visuospatial judgment: A clinical test. Archives of Neurology, 35, 364-367.

Brandt, J., \& Benedict, R. (2001). Hopkins verbal learning test-revised, psychological assessment resources, Lutz. Fl.

Burton, E. J., McKeith, I. G., Burn, D. J., Williams, E. D., \& O'Brien, J. T. (2004). Cerebral atrophy in Parkinson's disease with and without dementia: A comparison with Alzheimer's disease, dementia with Lewy bodies and controls. Brain: a Journal of Neurology, 127, 791-800.

Clarkson, M. J., Cardoso, M. J., Ridgway, G. R., Modat, M., Leung, K. K., Rohrer, J. D., et al. (2011). A comparison of voxel and surface based cortical thickness estimation methods. NeuroImage, 57, 856-865.

Compta, Y., Parkkinen, L., O'Sullivan, S. S., Vandrovcova, J., Holton, J. L., Collins, C., et al. (2011). Lewy-and Alzheimer-type pathologies in Parkinson's disease dementia: Which is more important? Brain: a Journal of Neurology, 134, 1493-1505.

Dale, A. M., Fischl, B., \& Sereno, M. I. (1999). Cortical surface-based analysis. I. Segmentation and surface reconstruction. NeuroImage, 9, 179-194.

Dujardin, K., Leentjens, A. F. G., Langlois, C., Moonen, A. J., Duits, A. A., Carette, A. S., et al. (2013). The spectrum of cognitive disorders in Parkinson's disease: A data-driven approach. Movement Disorders, 28, 183-189.

Dujardin, K., Moonen, A. J., Behal, H., Defebvre, L., Duhamel, A., Duits, A., et al. (2015). Cognitive disorders in Parkinson's disease: Confirmation of a spectrum of severity. Parkinsonism \& Related Disorders, 21, 1299-1305.

Duncan, G. W., Firbank, M. J., Yarnall, A. J., Khoo, T. K., Brooks, D. J., Barker, R. A., et al. (2016). Gray and white matter imaging: A biomarker for cognitive impairment in early Parkinson's disease? Movement Disorders, 31(1), 103-110.

Emre, M., Aarsland, D., Brown, R., Burn, D. J., Duyckaerts, C., Mizuno, Y., et al. (2007). Clinical diagnostic criteria for dementia associated with Parkinson's disease. Movement Disorders, 22, 1689-1707.

Fazekas, F., Chawluk, J. B., Alavi, A., Hurting, H. I., \& Zimmerman, R. A. (1987). MR signal abnormalities at $1.5 \mathrm{~T}$ in Alzheimer's dementia and normal aging. American Journal of Neuroradiology, 8, 421-426.

Fischl, B. (2012). Freesurfer. NeuroImage, 62, 774-781.

Fischl, B., Sereno, M. I., \& Dale, A. M. (1999). Cortical surface-based analysis. II: Inflation, flattening, and a surface-based coordinate system. NeuroImage, 9, 195-207.

Folstein, M. F., Folstein, S. E., \& McHugh, P. R. (1975). "Mini-mental state": A practical method for grading the cognitive state of patients for the clinician. Journal of Psychiatric Research, 12, 189-198.

Foltynie, T., Brayne, C. E., Robbins, T. W., \& Barker, R. A. (2004). The cognitive ability of an incident cohort of Parkinson's patients in the UK. The Campaign Study. Brain: a Journal of Neurology, 127, 550-560.

Gerrits, N. J. H. M., van Loenhoud, A. C., van den Berg, S. F., Berendse, H. W., Foncke, E. M. J., Klein, M., et al. (2016). Cortical thickness, surface area and subcortical volume differentially contribute to cognitive heterogeneity in Parkinson's disease. PLoS One, 11(2), e0148852.

Gibb, W., \& Lees, A. (1988). The relevance of the Lewy body to the pathogenesis of idiopathic Parkinson's disease. Journal of Neurology, Neurosurgery \& Psychiatry, 51, 745-752.

Goetz, C. G., Tilley, B. C., Shaftman, S. R., Stebbins, G. T., Fahn, S., Martinez-Martin, P., et al. (2008). Movement Disorder Society- sponsored revision of the Unified Parkinson's Disease Rating Scale (MDS-UPDRS): Scale presentation and clinimetric testing results. Movement Disorders, 23, 2129-2170.

Graves, R. E., Bezeau, S. C., Fogarty, J., \& Blair, R. (2004). Boston naming test short forms: A comparison of previous forms with new item response theory based forms. Journal of Clinical and Experimental Neuropsychology, 26, 891-902.

Hamilton, M. (1960). A rating scale for depression. Journal of Neurology, Neurosurgery, and psychiatry, 23, 56.

Harding, A., Broe, G., \& Halliday, G. (2002). Visual hallucinations in Lewy body disease relate to Lewy bodies in the temporal lobe. Brain: a Journal of Neurology, 125, 391-403.

Hely, M. A., Reid, W. G., Adena, M. A., Halliday, G. M., \& Morris, J. G. (2008). The Sydney multicenter study of Parkinson's disease: The inevitability of dementia at 20 years. Movement Disorders, 23, 837-844.

Hutton, C., Draganski, B., Ashburner, J., \& Weiskopf, N. (2009). A comparison between voxel-based cortical thickness and voxel-based morphometry in normal aging. NeuroImage, 48 , 371-380.

Janvin, C., Aarsland, D., Larsen, J. P., \& Hugdahl, K. (2003). Neuropsychological profile of patients with Parkinson's disease without dementia. Dementia and Geriatric Cognitive Disorders, 15, 126-131.

Jellinger, K., Seppi, K., Wenning, G., \& Poewe, W. (2002). Impact of coexistent Alzheimer pathology on the natural history of Parkinson's disease. Journal of Neural Transmission, 109, 329-339.

Kalaitzakis, M., \& Pearce, R. B. (2009). The morbid anatomy of dementia in Parkinson's disease. Acta Neuropathologica, 118, 587-598.

Kehagia, A. A., Barker, R. A., \& Robbins, T. W. (2010). Neuropsychological and clinical heterogeneity of cognitive impairment and dementia in patients with Parkinson's disease. The Lancet Neurology, 9, 1200-1213.

Kurosinski, P., Guggisberg, M., \& Götz, J. (2002). Alzheimer's and Parkinson's disease - Overlapping or synergistic pathologies? Trends in Molecular Medicine, 8, 3-5.

Lacadie, C. M., Fulbright, R. K., Rajeevan, N., Constable, R. T., \& Papademetris, X. (2008). More accurate Talairach coordinates for neuroimaging using non-linear registration. NeuroImage, 42, 717-725.

Lawton, M. P., \& Brody, E. M. (1969). Assessment of older people: Self-maintaining and instrumental activities of daily living. The Gerontologist 1969, 9, 179-186.

Leentjens, A. F. G., Dujardin, K., Pontone, G. M., Starkstein, S. E., Weintraub, D., \& Martinez-Martin, P. (2014). The Parkinson Anxiety Scale (PAS): Development and validation of a new anxiety scale. Movement Disorders, 29, 1035-1043.

Lerch, J. P., \& Evans, A. C. (2005). Cortical thickness analysis examined through power analysis and a population simulation. NeuroImage, 1, 163-173.

Levy, G. (2007). The relationship of Parkinson disease with aging. Archives of Neurology, 64, 1242-1246.

Litvan, I., Goldman, J. G., Tröster, A. I., Schmand, B. A., Weintraub, D., Petersen, R. C., et al. (2012). Diagnostic criteria for mild cognitive impairment in Parkinson's disease: Movement Disorder Society Task Force Guidelines. Movement Disorders, 27, 349-356.

Mattis, S. (1976). Mental status examination for organic mental syndrome in the elderly patient. In L. Bellak, \& T.L. (Eds.), Geriatric psychiatry (pp. 77-121). New York: Grune and Stratton.

Morris, J. C. (1993). The clinical dementia rating (Cdr): Current version and scoring rules. Neurology, 43, 2412-2414.

Nielsen, F.A. (2003). The Brede database: A small database for functional neuroimaging. 
Pereira, J. B., Svenningsson, P., Weintraub, D., Brønnick, K., Lebedev, A., Westman, E., et al. (2014). Initial cognitive decline is associated with cortical thinning in early Parkinson disease. Neurology, 82, 2017-2025.

Perry, E. K., Marshall, E., Kerwin, J., Smith, C. J., Jabeen, S., Cheng, A. V., et al. (1990). Evidence of a monoaminergiccholinergic Imbalance related to visual hallucinations in Lewy body dementia. Journal of Neurochemistry, 55, 1454-1456.

Reitan, R. (1992). Tmt. South Tucson: Trail Making Test a \& B.

Rektor, I., Svátková, A., Vojtíšek, L., Zikmundová, I., Vaníček, J., Király, A., et al. (2018). White matter alterations in Parkinson's disease with normal cognition precede grey matter atrophy. PLoS One, 5(1), 13.

Sabbagh, M. N., Adler, C. H., Lahti, T. J., Connor, D. J., Vedders, L., Peterson, L. K., et al. (2009). Parkinson's disease with dementia: Comparing patients with and without Alzheimer pathology. Alzheimer Disease and Associated Disorders, 23, 295.

Schaer, M., Cuadra, M. B., Tamarit, L., Lazeyras, F., Eliez, S., \& Thiran, J. P. (2008). A surface-based approach to quantify local cortical gyrification. IEEE Transactions on Medical Imaging, 27, 161-170.

Smith, A. (1982). Symbol Digits Modalities test. Los Angeles: Western Psychological Services.

Sockeel, P., Dujardin, K., Devos, D., Deneve, C., Destée, A., \& Defebvre, L. (2006). The Lille Apathy Rating Scale (Lars), a new instrument for detecting and quantifying apathy: Validation in Parkinson's disease. Journal of Neurology, Neurosurgery \& Psychiatry, 77, 579-584.
Song, S. K., Lee, J. E., Park, H. J., Sohn, Y. H., Lee, J. D., \& Lee, P. H. (2011). The pattern of cortical atrophy in patients with Parkinson's disease according to cognitive status. Movement Disorders, 26, 289-296.

Svenningsson, P., Westman, E., Ballard, C., \& Aarsland, D. (2012). Cognitive impairment in patients with Parkinson's disease: Diagnosis, biomarkers, and treatment. The Lancet Neurology, $11,697-707$

Tomlinson, C. L., Stowe, R., Patel, S., Rick, C., Gray, R., \& Clarke, C. E. (2010). Systematic review of levodopa dose equivalency reporting in Parkinson's disease. Movement Disorders, 25, 2649-2653.

Uc, E. Y., McDermott, M. P., Marder, K. S., Anderson, S. W., Litvan, I., Como, P. G., et al., Parkinson Study Group DATATOP Investigators. (2009). Incidence of and risk factors for cognitive impairment in an early Parkinson disease clinical trial cohort. Neurology, 73, 1469-1477.

Wechsler, D. (1986). Wais-R: Wechsler adult intelligence scale, revised.

Weil, R. S., Costantini, A. A., \& Schrag, A. E. (2018). Mild cognitive impairment in Parkinson's disease-What is it? Current Neurology and Neuroscience Reports, 18(4), 17.

Weintraub, D., Doshi, J., Koka, D., Davatzikos, C., Siderowf, A. D., Duda, J. E., et al. (2011). Neurodegeneration across stages of cognitive decline in Parkinson disease. Archives of Neurology, 68, 1562-1568.

Yarnall, A. J., Breen, D. P., Duncan, G. W., Khoo, T. K., Coleman, S. Y., Firbank, M. J., et al. (2014). Characterizing mild cognitive impairment in incident Parkinson disease the ICICLE-PD Study. Neurology, 82, 308-316. 